\title{
Combinations of Tumor and Treatment Parameters Are More Discriminative for Prognosis Than the Present TNM System in Rectal Cancer
}

\author{
Iris D. Nagtegaal and Marleen J.E.M. Gosens, Department of Pathology, Radboud University Medical Center, Nijmegen, \\ the Netherlands \\ Corrie A.M. Marijnen, Department of Radiotherapy, Netherlands Cancer Institute, Amsterdam, the Netherlands \\ Harm J. Rutten, Department of Surgery, Catharina Hospital, Eindhoven, the Netherlands \\ Cornelis J.H. van de Velde, Department of Surgery, Leiden University Medical Center, Leiden, the Netherlands \\ J. Han J.M. van Krieken, Department of Pathology, Radboud University Medical Center, Nijmegen, the Netherlands
}

The treatment of rectal cancer has changed radically during the last decade. The introduction of the surgical technique total mesorectal excision (TME) worldwide has resulted in a decline of local recurrence rate because more tumors were excised completely, along with the excision of regional metastatic disease in the mesorectal fat. In addition, the role of neoadjuvant treatment either by radiotherapy or by radiochemotherapy has been established during the last 10 years. In Europe, overwhelming evidence has been gathered from large randomized trials (Total Mesorectal Excision trial [TME], Swedish Rectal Cancer Trial, and Cancer Research UK [CR07]), ${ }^{1-3}$ with a total number of 4,427 patients, showing that for primarily resectable rectal cancer, short-term preoperative radiotherapy ( 5 Gy daily for 5 days) resulted in local recurrence rates lower than $5 \%$, especially in combination with TME surgery. For locally advanced tumors, long-term radiotherapy (approximately $50 \mathrm{~Gy}$ ) in combination with neoadjuvant chemotherapy is the treatment of choice. ${ }^{4,5}$

The combination of the above-mentioned changes in therapy results in improved prognosis of patients with rectal cancer, especially with respect to local recurrence, but these advances have not yet been included in staging of rectal carcinoma. In fact, these innovations in therapy call for a change in the approach of staging. Because of the application of neoadjuvant therapy, both the function of staging systems and the factors used for staging have changed, which complicates the current practice.

Initially, postoperative pathologic staging was used for the prediction of prognosis as well as for the indication of adjuvant therapy. At present, clinical staging determines whether and which preoperative therapy should be applied, and postoperative staging is used to evaluate the effects of therapy in addition to the above-mentioned goals. The consequence of these changes is a divergence between clinical TNM and pathologic (p) TNM. Moreover, the current pTNM is essentially different from the pTNM of the last century. Still, the staging system for rectal cancer uses the same rules as Cuthbert Dukes proposed in $1932 .{ }^{6}$

\section{IMPACT OF NEOADJUVANT THERAPY}

Long-term radiotherapy and chemoradiotherapy schemes are aimed at tumor downstaging to facilitate complete surgical removal. Pronounced changes in tumor histology are observed in the operation specimen, and are indicative of tumor response or regression. In many of these cases, the $\mathrm{pT}$ stage is lowered compared with the initial cT stage, but it is not clear which of these two is the best predictor for prognosis. The current guidelines of the American Joint Committee on Cancer TNM staging systems ${ }^{7,8}$ acknowledge preoperative treatment by adding the prefix $y$, but the clinical consequences are not clear.

The ypT stage can be used as a measurement for tumor downstaging, however, after locally advanced tumors are removed, tumor remnants might be left behind in the surrounding tissue, resulting in inadequate determination of T stage. Moreover, there is a large variability between the pT3 tumors with regard to tumor load. Alternatively, response can be indicated by determining the grade of tumor regression. Various systems have been suggested to grade tumor regression, ${ }^{9-11}$ but the majority are not able to demonstrate a relation with prognosis. In addition, reproducibility of regression grading is poor. ${ }^{11,12}$

Given that the goal of long-term neoadjuvant therapy is the facilitation of surgical removal, we suggest inclusion of surgery-related factors in the staging after this kind of treatment.

\section{SURGICAL FACTORS IN THE 21st CENTURY}

The recognition of TME as a superior surgical technique is preceded by the recognition of circumferential margin (CRM) involvement as the best prognostic factor, not only for local recurrence, but also for development of metastases and for survival. A recent review with data of more than 17,500 patients (unpublished data: Nagtegaal ID and Quirke P, "What role for the circumferential margin in the modern treatment of rectal cancer," 2007) demonstrated that the prognostic 
value of an involved CRM for local recurrence is even stronger after neoadjuvant therapy (hazard ratio, 6.3; 95\% CI, 3.6 to 16.7 versus hazard ratio, $2.2 ; 95 \% \mathrm{CI}, 1.5$ to 3.2 without neoadjuvant therapy).

A positive CRM after surgery can be caused by various factors, the most important of which are suboptimal quality of surgery, aggressive tumor growth, therapy resistance, and inadequate preoperative imaging. The quality of surgery is analyzed by the assessment of plane of resection. This is correlated with both local recurrence and overall survival, and its value has been confirmed recently in another large multicenter trial. ${ }^{13}$ The finding that CRM involvement can predict the development of distant metastases as well as survival may suggest that aggressive tumor growth is most important. ${ }^{14}$ However, the fact that a positive CRM due to poor-quality surgery also is correlated with survival ${ }^{14}$ indicates that for prognosis, the cause of margin involvement does not seem to matter. ${ }^{14}$

\section{STAGING SYSTEMS: WHERE SHOULD WE GO?}

In the era of neoadjuvant therapy, the existing staging systems are suboptimal. There is a need for the implementation of treatmentrelated factors, which will improve both staging and prediction of prognosis. ${ }^{15}$ The result of treatment is one of the most relevant features for predicting final outcome; therefore, modern staging systems should take both tumor and treatment factors into account. The incorporation of these factors, of course, should be based on evidence. Before we can propose a new staging system, we have to address the following questions: Which factors can predict prognosis reliably? Are these factors generally applicable? Can these factors be assessed in a reliable and simple way? Is there a combination of factors that divides patients adequately in large, homogeneous groups with highly divergent survival curves?

\section{Which Factors Can Predict Prognosis Reliably?}

First, we have to question the value and reliability of established tumor factors such as invasion depth and lymph node status in the current situation. As mentioned, the reliability and relevance of ypT is questionable. The presence of lymph node metastases after neoadjuvant therapy is still a major prognostic factor. ${ }^{16-18}$ However, an unknown number of node-negative patients will have had positive nodes that are sterilized by neoadjuvant therapy. Therefore, ypN0 consists of a heterogeneous group of patients who were initially node negative and patients whose metastatic tumors responded well to treatment. Although the meaning of ypN0 might be different from that of $\mathrm{pN} 0$, the prognostic impact is still applicable. However, a multivariate analysis of 182 patients ${ }^{18}$ suggests that after neoadjuvant therapy, CRM is more important for prognosis than lymph node involvement. Given that neoadjuvant therapy is mainly aimed on local control, at present, we can leave the presence of metastatic disease (TNM IV) out of this discussion.

Treatment-related factors are CRM, tumor regression, and quality of surgery. The results of tumor regression grading are variable and no consistent relation with prognosis has been demonstrated. Moreover, four different studies including a total of 490 patients demonstrated the superiority of CRM assessment above regression grading. ${ }^{12,19-21}$ Quality of surgery evaluation in two independent randomized trials demonstrates prognostic value for both local recur-

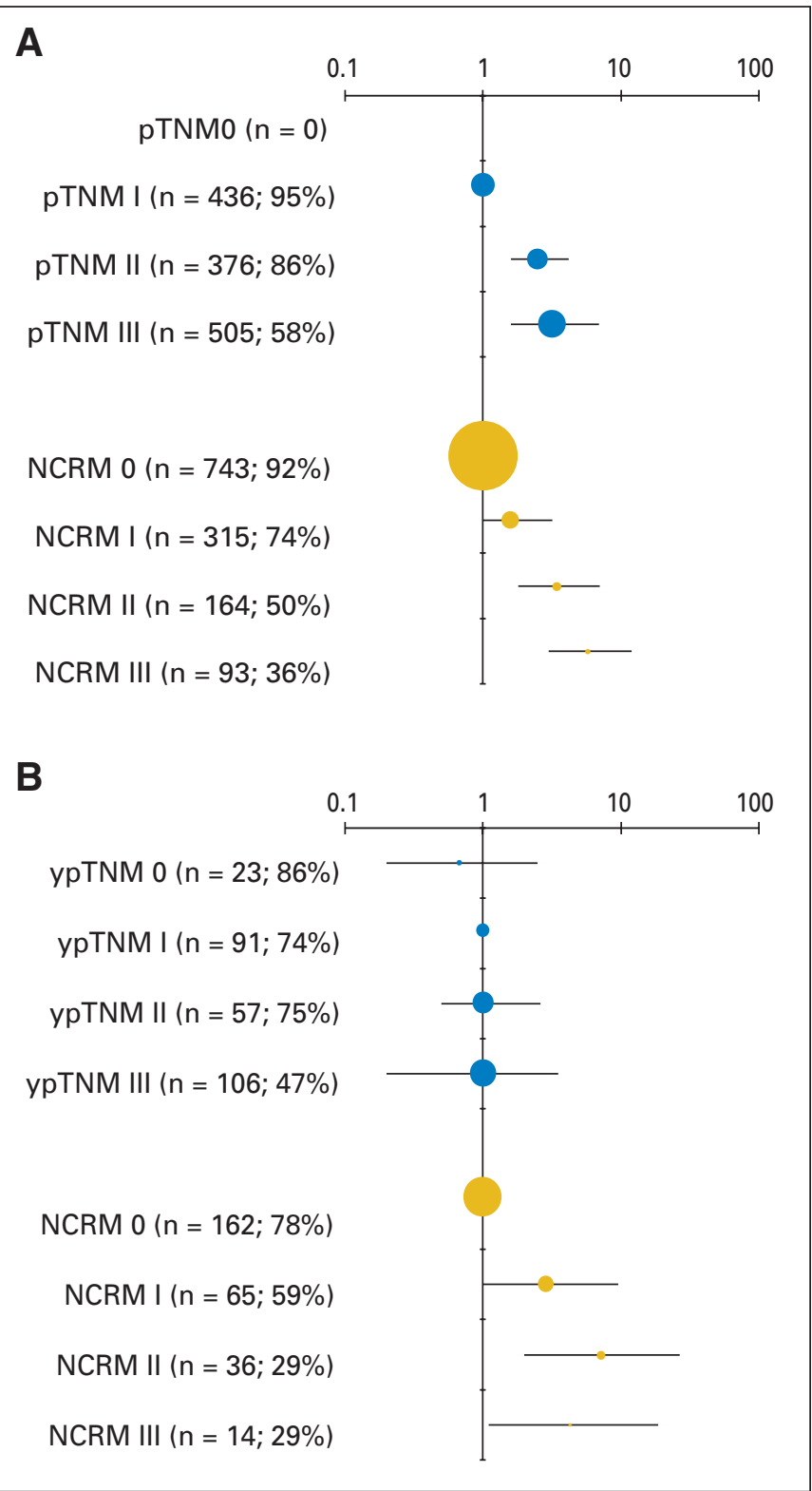

Fig 1. Hazard ratio and $95 \%$ confidence interval of TNM staging versus a new staging method based on nodal and circumferential margin status (NCRM). (A) TME trial ( $n=1530$; follow-up 67 months). (B) Polish rectal cancer trial $(n=316$; follow-up 48 months). y, preoperative treatment; p, pathologic.

rence and survival. ${ }^{13,14}$ However, CRM involvement is more important than plane of surgery. ${ }^{14}$

Finally, there are many biomarkers described, but none of them have reached the standard assessment of rectal cancer specimens and therefore remain beyond the scope of this commentary.

\section{Are These Factors Generally Applicable?}

Although preoperative neoadjuvant therapy will be applied in most occurrences of rectal cancer, some patients will undergo surgery right away. The new staging should be applicable in all situations. Tumor invasion, lymph node metastases, CRM involvement, and quality of surgery can be evaluated with and without neoadjuvant therapy and in any laboratory of pathology. One could argue that this is the case for tumor regression as well, and that without therapy there 
will be no regression. As a result, although the absence of regression after therapy may indicate poor prognosis, the absence of regression without therapy has no meaning at all.

\section{Can These Factors Be Determined in a Reliable and Simple Way?}

The stage of the tumor is relatively simple to determine provided that an adequate sampling of the tumor area is performed. Especially for the determination of ypT0 (complete regression), a standardized protocol is required. Careful examination of the resection specimen will reveal possible involvement of the circumferential margin and presence of lymph node metastasis. Detailed protocols are available. ${ }^{22,23}$

Determination of tumor regression is much more difficult and reproducibility studies show $\kappa$ values as low as $0.30 .{ }^{12}$ One of the reasons is that there is no consensus about the definitions that should be used, apart from the definition of complete response. It is disappointing that to date, none of the reported studies used this definition.

\section{Is There a Combination of Factors That Adequately Divides Patients in Large, Homogeneous Groups With Highly Divergent Survival Curves?}

In a recent study ${ }^{24}$ based on the data from a randomized clinical trial, ${ }^{1}$ we demonstrated in a multivariate model that CRM rather than pT stage is important for the prognosis of rectal cancer. This is true for patients without preoperative treatment as well as for patients treated with short-term preoperative radiotherapy. On the basis of these findings, we designed a staging system including both CRM (treatmentrelated factor) and nodal status (tumor-related factor; Fig 1). Using this system, we created highly divergent survival curves, with a small group of patients with a poor prognosis $(n=93 ; 7 \% ; 37 \% 5$-year survival) and a large group of patients with a good prognosis $(\mathrm{n}=753$; 57\%; 92\% 5-year survival). We confirmed our findings in an independent data set derived from another randomized trial ${ }^{25}$ (Fig 1B). In this group of patients (with short-term and long-term neoadjuvant therapy) the new staging system performed significantly better than TNM.

In conclusion, in modern staging of cancer there should be an important place for treatment-related factors, given that the result of treatment is one of the most important prognostic factors. For rectal cancer we demonstrated that in the era of neoadjuvant therapy, free circumferential resection margins are more important than the classic factor of invasion depth, and that incorporation of this factor in staging systems leads to better prediction of prognosis and selection of patients.

\section{AUTHORS' DISCLOSURES OF POTENTIAL CONFLICTS OF INTEREST Although all authors completed the disclosure declaration, the following author or immediate family members indicated a financial interest. No conflict exists for drugs or devices used in a study if they are not being evaluated as part of the investigation. For a detailed description of the disclosure categories, or for more information about ASCO's conflict of interest policy, please refer to the Author Disclosure Declaration and the Disclosures of Potential Conflicts of Interest section in Information for Contributors. \\ Employment: N/A Leadership: N/A Consultant: N/A Stock: N/A Honoraria: N/A Research Funds: Corrie A.M. Marijnen, Roche Testimony: N/A Other: N/A}

\section{AUTHOR CONTRIBUTIONS}

Conception and design: Iris D. Nagtegaal, Corrie A.M. Marijnen, J.H.J.M van Krieken
Manuscript writing: Iris D. Nagtegaal, Marleen J.E.M. Gosens, Corrie A.M. Marijnen, Harm J. Rutten, Cornelis J.H. van de Velde, J.H.J.M van Krieken

Final approval of manuscript: Iris D. Nagtegaal, Marleen J.E.M. Gosens, Corrie A.M. Marijnen, Harm J. Rutten, Cornelis J.H. van de Velde, J.H.J.M van Krieken

\section{REFERENCES}

1. Kapiteijn E, Marijnen CAM, Nagtegaal ID, et al: Preoperative radiotherapy combined with total mesorectal excision for resectable rectal cancer. N Engl J Med 345:638-646, 2001

2. Swedish Rectal Cancer Trial: Improved survival with preoperative radiotherapy in resectable rectal cancer. N Engl J Med 336:980-987, 1997

3. Sebag-Montefiore D, Steele R, Quirke $P$, et al: Routine short course preoperative radiotherapy or selective postoperative chemoradiotherapy for resectable rectal cancer? Preliminary results of the MRC CR07 randomized trial. $J$ Clin Oncol 24:148s, 2006 (abstr 3511)

4. Gerard JP, Conroy T, Bonnetain F, et al: Preoperative radiotherapy with or without concurrent fluorouracil and leucovorin in T3-4 rectal cancers: Results of FFCD 9203. J Clin Oncol 24:4620-4625, 2006

5. Bosset JF, Collette L, Calais, et al: Chemotherapy with preoperative radiotherapy in rectal cancer. N Engl J Med 355:1114-1123, 2006

6. Dukes CE: The classification of cancer of the rectum. J Pathol Bacteriol 35:323-332, 1932

7. Sobin LH, Wittekind C: TNM Classification of Malignant Tumours (ed 6). New York, NY, John Wiley, 2002

8. Greene FL, Page D, Fleming ID, et al: AJCC Staging Handbook (ed 6). New York, NY, Springer, 2002

9. Rodel C, Martus P, Papadoupolos T: Prognostic significance of tumour regression after preoperative chemoradiation for rectal cancer. J Clin Oncol 23:8688-8696, 2006

10. Bouzourene H, Bosman FT, Seelentag $W$, et al: Importance of tumor regression assessment in predicting the outcome in patients with locally advanced rectal carcinoma who are treated with preoperative radiotherapy. Cancer 94:1121-1130, 2002

11. Ryan R, Gibbons D, Hyland JM, et al: Pathological response following long-course neoadjuvant chemoradiotherapy for locally advanced rectal cancer. Histopathology 47:141-146, 2005

12. Gosens MJEM, Van Krieken JH, Rutten $H$, et al: A critical appraisal of therapy induced tumor regression in rectal carcinoma. Ann Oncol 17:P62, 2006

13. Quirke P, Sebag-Montefiore D, Steele R, et al: Local recurrence after rectal cancer resection is strongly related to the plane of surgical dissection and is further reduced by preoperative short course radiotherapy: Preliminary results of the MRC CR07 trial. J Clin Oncol 24:149s, 2006 (abstr 3512)

14. Nagtegaal ID, van de Velde CJH, van der Worp E, et al: Macroscopic evaluation of rectal cancer resection specimen: Clinical significance of the pathologist in quality control. J Clin Oncol 20:1729-1734, 2002

15. Jeruss JS, Mittendorf EA, Tucker SL, et al: Should pathological response to neoadjuvant therapy be incorporated into the AJCC staging system for breast cancer? Breast Cancer Res Treat 100:s259, 2006

16. Das $P$, Skibber JM, Rodriguez-Bigas MA, et al: Clinical and pathologic predictors of locoregional recurrence, distant metastasis, and overall survival in patients treated with chemoradiation and mesorectal excision for rectal cancer. Am J Clin Oncol 29:219-224, 2006

17. Kim NK, Baik SH, Seong JS, et al: Oncologic outcomes after neoadjuvant chemoradiation followed by curative resection with tumor-specific mesorectal excision for fixed locally advanced rectal cancer: Impact of postirradiated pathologic downstaging on local recurrence and survival. Ann Surg 244:1024-1030, 2006

18. Beresford M, Glynne-Jones $R$, Richman $P$, et al: The reliability of lymphnode staging in rectal cancer after preoperative chemoradiotherapy. Clin Oncol $(R$ Coll Radiol) 17:448-455, 2005

19. Mawdsley S, Glynne-Jones R, Grainger J, et al: Can histopathologic assessment of circumferential margin after preoperative pelvic chemoradiotherapy for T3-T4 rectal cancer predict for 3-year disease-free survival? Int J Radiat Oncol Biol Phys 63:745-752, 2005

20. Bouzourene $H$, Bosman FT, Matter $M$, et al: Predictive factors in locally advanced rectal cancer treated with preoperative hyperfractionated and accelerated radiotherapy. Hum Pathol 34:541-548, 2003

21. Machiels JP, Aydin S, Bonny MA, et al: What is the best way to predict disease-free survival after preoperative radiochemotherapy for rectal cancer 
patients: Tumor regression grading, nodal status, or circumferential resection margin invasion? J Clin Oncol 24:1319, 2006

22. Quirke P, Dixon MF: How I do it: The prediction of local recurrence in rectal adenocarcinoma by histopathological examination. Int J Colorectal Dis 3:127-131, 1988

23. Quirke P, Morris E: Reporting colorectal cancer. Histopathology 50:103112,2007
24. Gosens $M$, van Krieken JHJM, Marijnen, CAM, et al: Improvement of staging by combining tumour and treatment parameters: The value for prognostification in rectal cancer. Clin Gastroenterol Hepatol (in press)

25. Bujko K, Nowacki MP, Nasierowska-Guttmejer A, et al: Sphincter preservation following preoperative radiotherapy for rectal cancer: Report of a randomised trial comparing short-term radiotherapy vs. conventionally fractionated radiochemotherapy. Radiother Oncol 72:15-24, 2004

\section{Acknowledgment}

I.D.N. is a fellow of the Dutch Cancer Society. 